\title{
579 EXPLOITING TUMOR NEOANTIGEN-TARGETED IMMUNOTHERAPY IN IMMUNOLOGICALLY HOT VERSUS COLD MURINE LUNG CANCER MODELS
}

${ }^{1}$ Changbo Sun*, ${ }^{1}$ Koji Nagaoka, ${ }^{2}$ Yukari Kobayashi, ${ }^{1} J u n$ Nakajima, ${ }^{1}$ Kazuhiro Kakimi.

${ }^{1}$ University of Tokyo Hospital, Tokyo, Japan; ${ }^{2}$ University of Tokyo, Bunkyo-ku, Japan

Background The treatment of non-small cell lung cancer has been altered by immune-checkpoint therapy in the last 10 years. However, many patients still do not respond to it or, eventually, the disease progresses. One of the factors leading to primary resistance to ICI is the "cold" tumor, characterized by the absence of $\mathrm{T}$ cell infiltration and not sufficiently primed for immune recognition. Here, we compared the neoantigen-based immunotherapy in two lung cancer cell lines: $\mathrm{T}$ cell-inflamed ASB-XIV and non-T cell-inflamed LLC1.

Methods Whole-exome and RNA sequencing were performed to identify neoantigens. First, MHC binding scores of expressed mutated peptides $(\mathrm{FPKM}>1)$ were estimated to select candidate neoantigens. Next, the immunogenicity of predicted mutated peptides was investigated. Then, the anti-tumor effect of immunogenic mutated peptides was accessed. Finally, immunosuppressive molecules in the non-T cell inflamed tumor microenvironment were investigated and targeted for effective treatment.

Results Inflamed ASB-XIV tumors were sensitive to ICI, while non-inflamed LCC1 tumors were resistant to ICI. Using ASBXIV-specific CTLs, we screened the panel of candidate neoantigen peptides and identified Phf3 N1867K mutated peptide as neoepitope. Mutated Phf3 peptide-pulsed DCs induced peptide-specific CD8 $+\mathrm{T}$ cells and inhibited the ASB-XIV tumor growth in the prophylactic and therapeutic setting. Furthermore, adoptive transfer of $\mathrm{mPhf3}$-specific CTLs also eradicated the ASB-XIV tumors. In the case of LLC1, twenty-five out of 132 short mutated peptides induced peptide-specific CD8 $+\mathrm{T}$ cell response, but they could not inhibit the LLC1 tumor growth. DC vaccines pulsed with long peptides (LP) induced both CD4+ and CD8 + T cell responses ex vivo. Of them, DC pulsed with LP82 partly delayed the LLC1 growth in vivo. By RNA-Seq, CD38 was highly expressed in LLC1. Thereby, an anti-CD38 antibody was administered in LLC1bearing mice immunized with DC pulsed with LP82. The tumor growth was suppressed in the combination treatment, partly because CD38 blockade decreased regulatory $\mathrm{T}$ cells in the tumor.

Conclusions Responses to neoantigen-targeted immunotherapy preferentially observed in $\mathrm{T}$ cell-inflamed tumors. Regulation of immunosuppressive tumor microenvironment is required to make neoantigen-targeted immunotherapy effective in noninflamed tumors.

http://dx.doi.org/10.1136/jitc-2021-SITC2021.579 